\title{
The Application Programmer Interface (API) in Modern Cartography: Development and Prospects
}

\author{
Michael P. Peterson* \\ *University of Nebraska at Omaha, Geography/Geology, Omaha, NE, 68182; mpeterson@unomaha.edu
}

Keywords: API, Online Mapping, Cloud Tools, Tile-based Mapping, MultiScale Pannable (MSP) mapping

\begin{abstract}
:
Application Programmer Interfaces have been a part of the cartographic landscape since 2006 with the introduction of the Google Maps API. Essentially a library of code that provides access to a variety of mapping functions, APIs have since been the basis of online, tile-based, Multiscale Pannable (MSP) mapping. While the Google Maps API is still the most widely used with more than 4.6 billion websites embedding a Google Map, a variety of other mapping APIs have been introduced primarily to circumvent Google's pricing structure.

The cost for using Google Maps on websites has changed over the years. From 2005 to 2011, the use of Google Maps was free no matter how many maps were referenced by a website. From then to 2016, Google limited map downloads to 25,000 map loads a day for 90 consecutive days. In 2016, the 90 consecutive days was removed so the number of maps downloaded could not exceed 25,000 on any given day. If it did, the website developer needed to register their site and pay a fee for maps produced over this limit.

In April 2018, Google announced the launch of a new name for the Google Maps API - Google Maps Platform - and a new pricing plan. A free tier continues to be offered through a US \$200 monthly credit but now requires creating an account and entering a credit card number. In this new online platform, it is still possible to use the Google Maps API without incurring any cost by limiting the number of daily downloads so that the monthly quota of 28,000 map downloads is not exceeded.

The problem is not the use of the Google Maps API but the Google map tiles. The look of maps from Google and the associated interface have become so popular that users avoid using other kinds of maps - even those from Apple. Users complain that the tiles from other vendors implement a different color scheme or highlight different features. They also complain that these maps appear more slowly. While other APIs can use Google Map tiles, including the popular Leaflet API, their use is still subject to the same pricing structure.

With the help of the Internet, maps from Google have become the standard maps. All other renditions of the world are seen to be inferior and not worthy of examination. They are simply interesting oddities. While some can adapt to alternative representations, most choose not to. This Google Map phenomena is examined along with lessons from the historical progression of online mapping.
\end{abstract}

